\title{
Oscillations in seasonal SIR models with saturated treatment
}

\author{
L. Rocío González-Ramírez ${ }^{a, b}$, Osvaldo Osuna ${ }^{a *}$, \\ Geiser Villavicencio-P Ulido ${ }^{c}$ \\ ${ }^{a}$ Universidad Michoacana de San Nicolás de Hidalgo, Instituto de Física y \\ Matemáticas, Morelia, México. \\ ${ }^{b}$ Conacyt, D.F., México. \\ ${ }^{c}$ Universidad Autónoma Metropolitana Unidad Lerma, Departamento de Ciencias \\ Ambientales, Estado de México, México.
}

\begin{abstract}
In this work, we give some conditions for the existence of periodic orbits for a Susceptible-Infectious-Recovered (SIR) model with seasonal saturated incidence functions and saturated treatment rate. We use LeraySchauder degree theory to prove the existence of periodic orbits.

Keywords: Leray-Schauder degree, SIR models, periodic orbits, reproductive number.

MSC2010: 37J45, 34C25, 92D30, 34D23.

\section{Oscilaciones en modelos SIR estacionales con tratamiento saturado}

Resumen. En este trabajo presentamos condiciones suficientes para la existencia de soluciones periódicas en modelos epidemiológicos estacionales de tipo SIR con funciones de incidencia y de tratamiento saturados. Utilizamos la teoría de grado de Leray-Schauder para establecer la existencia de órbitas periódicas en tales modelos.

Palabras clave: Grado de Leray-Schauder, modelo SIR, órbitas periódicas, número reproductivo básico.

\section{Introduction}

Based on the fact that many infectious diseases exhibit periodic fluctuations, such as pertussis, rubeola, influenza, polio, chickenpox, mumps, rabies, etc., we consider a

\footnotetext{
*E-mail: osvaldo@ifm.umich.mx

Received: 29 January 2016, Accepted: 11 May 2016.

To cite this article: L.R. González-Ramírez, O. Osuna, G. Villavicencio-Pulido, Oscillations in seasonal

SIR models with saturated treatment, Rev. Integr. Temas Mat. 34 (2016), No. 2, 125-131.
} 
Susceptible-Infectious-Recovered (SIR) model with saturated and periodic incidence rate and saturated treatment function

$$
\begin{aligned}
S^{\prime} & =\Lambda-d S-\beta(t) S I /(1+k I), \\
I^{\prime} & =\beta(t) S I /(1+k I)-(d+\epsilon+\mu) I-\gamma I /\left(1+\alpha I^{n}\right), \\
R^{\prime} & =\mu I+\gamma I /\left(1+\alpha I^{n}\right)-d R, n \in \mathbb{N} \cup\{0\},
\end{aligned}
$$

where $S, I$ and $R$ are the fractions of the population representing susceptible, infective and recovered individuals, respectively.

All of the parameters are positive constants. The natural death rate and birth rate are assumed to be equal, and denoted by $d, \epsilon$ is the disease-related death rate. It is assumed that the infected population recovers at a rate of $\mu$ and joins the recovered class. The interactions between susceptible and infected individuals is modeled by the incidence function $\beta(t) S I /(1+k I)$, with $\beta$ a non constant function (several different incidence rates of this type with $\beta$ constant have been proposed by authors, for example: [2], [7] and [11]). The use of a periodic incidence function can account for the variability of diseases according to climate seasons, school calendars, etc.

We also consider a saturated treatment rate $T(I)=\gamma I /\left(1+\alpha I^{n}\right)$ to describe the effect of the infected individuals being delayed for treatment due to the inhibition effect from the behavioral change of the susceptible individuals when their number increases or from the crowding effect of the infective individuals; this class of treatment was considered in [6], [8] and [12]. In the treatment function, $\alpha$ is a parameter to measure the extent of the delaying treatment to the infected and $\gamma$ is the cure rate. The parameter $n$ yields different treatment strategies, so, if $n=0$ the rate of treatment is constant, that is, each infectious individual has an equal chance of being treated. When $n=1$ the function $T(I)$ is a function of saturation; thus, when the treatment capacity of a community is limited, then from certain value of $I(t)$ the total treatment rate is almost constant. Now, if $n>1$ the treatment $T(I)$ reaches a maximum at $I^{*}=\frac{1}{\alpha^{\frac{1}{n}}(n-1)^{\frac{1}{n}}}$; thus, the treatment rate is small at the beginning of the outbreak, after the treatment rate will be increased with the improving of the treatment conditions, for example including effective medicines, skillful techniques, etc., reaching a maximum. At last, from the critical value $I^{*}$ total treatment rate begins to decrease. This type of treatment rates can model scenarios where infected people accumulate critical supplies, or the number of workers health is diminished because some are victims of the infection. That was certainly the case in the influenza pandemic of 1918. Another scenario is that treatment centers could be filled or lost, leaving a greater portion of non treated spreading the disease infected more easily, etc.

\section{Existence results}

At any given time, $t$, the total population size $N=S+I+R$ is variable with $N^{\prime}=$ $\Lambda-d N-\epsilon I$. In the absence of disease, the population size $N$ approaches the carrying capacity $\Lambda / d$. The differential equation for $N$ implies that solutions of (1) starting in the positive orthant $\mathbb{R}_{+}^{3}$ either approach, enter, or remain in the subset of $\mathbb{R}^{3}$ defined by

$$
\Sigma_{3}:=\{(S, I, R) \mid S \geq 0, I \geq 0, R \geq 0, S+I+R \leq \Lambda / d\}
$$

[Revista Integración 
Thus, it suffices to consider solutions in the invariant set $\Sigma_{3}$. Moreover, since the third equation is independent of the first two equations in system (1), it can be ignored, so we can study the following reduced model:

$$
\left\{\begin{array}{c}
S^{\prime}=\Lambda-d S-\beta(t) S I /(1+k I), \\
I^{\prime}=\beta(t) S I /(1+k I)-(d+\epsilon+\mu) I-\gamma I /\left(1+\alpha I^{n}\right) .
\end{array}\right.
$$

We restrict our attention to the region $\Sigma_{2}:=\left\{(S, I) \in \mathbb{R}^{2}: 0<S, 0<I, S+I<\Lambda / d\right\}$. The existence of periodic orbits for (2) implies the existence of periodic solution for system (1).

It is easy to see that the system (2) has an infection-free equilibrium state $\left(S_{0}, I_{0}\right)=$ $(\Lambda / d, 0)$.

The basic reproductive number $\mathcal{R}_{0}$ of model (2), i.e., the average number of secondary cases produced by a single infective introduced into an entirely susceptible population is given (see [9]), when $\beta$ is constant, for the system (2); a direct calculation yields

$$
\mathcal{R}_{0}:=\frac{\beta S_{0}}{d+\epsilon+\mu+\gamma} .
$$

Motivated by this, we define $\mathcal{R}_{0}$ for system (2) as follows:

$$
\mathcal{R}_{0}:=\frac{\bar{\beta} \Lambda / d}{d+\epsilon+\mu+\gamma}
$$

where $\bar{\beta}:=\frac{1}{T} \int_{0}^{T} \beta(t) d t$.

Now we state our main result

Theorem 2.1. If $\mathcal{R}_{0}>1+k \Lambda / d$, then the system (2) admits a non-trivial periodic solution.

$\mathrm{Li}$ and coworkers [6] studied the existence of periodic solutions of model (1) when $n=1$, by using Mawhin's continuation theorem [3]. The existence of periodic orbits of some epidemiological models with $\Lambda=d$ and $k=\gamma=0$ is analyzed in [4] and [5].

We carry out the proof of this theorem in two parts. First taking $k=\alpha=0$. We write

$$
\beta(t)=\bar{\beta}+\beta_{0}(t), \text { where } \int_{0}^{T} \beta_{0}(t) d t=0 .
$$

For $\lambda \in[0,1]$ we define the homotopy

$$
\left\{\begin{array}{c}
S^{\prime}=\Lambda-d S-\beta_{\lambda} I S \\
I^{\prime}=\beta_{\lambda} I S-(d+\epsilon+\mu+\gamma) I
\end{array}\right.
$$

where $\beta_{\lambda}:=\bar{\beta}+\lambda \beta_{0}(t)$.

Vol. 34, No. 2, 2016] 
To show the existence of a positive periodic solution, we shall use the Leray-Schauder degree theory [1]. So, we need to reformulate the problem in a functional setting. We consider the Banach spaces

$$
\mathcal{C}^{l}:=\left\{(S, I): S, I \in C^{l}(\mathbb{R}, \mathbb{R}), S(t+T)=S(t), I(t+T)=I(t)\right\}, l=0,1 .
$$

Let $L: \mathcal{C}^{1} \rightarrow \mathcal{C}^{0}$ and $N_{\lambda}: \mathcal{C}^{0} \rightarrow \mathcal{C}^{0}$ be the operators given by

$$
L(S, I):=\left(S^{\prime}+d S, I^{\prime}+(d+\epsilon+\mu) I\right),
$$

and

$$
N_{\lambda}(S, I):=\left(\Lambda-\beta_{\lambda} I S, \beta_{\lambda} I S-\gamma I\right) .
$$

Since $L$ is invertible we define

$$
F_{\lambda}(S, I):=(S, I)-L^{-1} \circ N_{\lambda}(S, I) .
$$

Since $\mathcal{C}^{1}$ is compactly embedded in $\mathcal{C}^{0}$, we can think of $L^{-1}$ as going from $\mathcal{C}^{0}$ to $\mathcal{C}^{0}$, therefore $L^{-1} \circ N_{\lambda}: \mathcal{C}^{0} \rightarrow \mathcal{C}^{0}$ is a compact operator. In a similar fashion, we can consider $F_{\lambda}: \mathcal{C}^{0} \rightarrow \mathcal{C}^{0}$. Thus, (4) is a functional reformulation of problem (3); in particular, periodic solutions of (3) correspond to zeroes of $F_{\lambda}$.

We consider the open sets $D:=\left\{(S, I) \in \mathcal{C}^{0}: 0<S, 0<I, S+I<\Lambda / d\right\}$ and

$$
U:=\left\{(S, I) \in D: \min _{[0, T]} S(t)<r(\Lambda / d)\right\}
$$

for a fixed $0<r<1$. Note that we can adapt the results of the work developed in [5]; thus we get

Proposition 2.2. If $\mathcal{R}_{0}>1$, then there is at least one T-periodic orbit of (3) whose components are positive.

In particular, we obtain that $\operatorname{deg}\left(F_{1}, U\right) \neq 0$.

To analyze the more general case (2), we consider a second homotopy. For $\tau \in[0,1]$ we define the homotopy

$$
\left\{\begin{array}{c}
S^{\prime}=\Lambda-d S-\beta(t) S I /(1+\tau k I), \\
I^{\prime}=\beta(t) S I /(1+\tau k I)-(d+\epsilon+\mu) I-\gamma I /\left(1+\tau \alpha I^{n}\right) .
\end{array}\right.
$$

To reformulate our problem in a functional context, we consider the operator $N_{\lambda}: \mathcal{C}^{0} \rightarrow$ $\mathcal{C}^{0}$ given by

$$
M_{\lambda}(S, I):=\left(\Lambda-\beta S I /(1+\tau k I), \beta S I /(1+\tau k I)-\gamma I /\left(1+\tau \alpha I^{n}\right)\right),
$$

and we define

$$
G_{\tau}(S, I):=(S, I)-L^{-1} \circ M_{\tau}(S, I) .
$$

As already discussed, we can consider $G_{\tau}: \mathcal{C}^{0} \rightarrow \mathcal{C}^{0}$. Thus, (6) is a functional reformulation of problem (5); in particular, periodic solutions of (5) correspond to zeroes of $G_{\tau}$.

Recall that the existence of a solution for $G_{1}$ in $U$ via Leray-Schauder degree is guaranteed if $\operatorname{deg}\left(G_{0}, U\right) \neq 0$ and $G_{\tau}$ is an admissible homotopy, i.e., $0 \notin G_{\tau}(\partial U), \forall \tau \in[0,1]$. The next result says that $G_{\tau}$ is admissible. 
Lemma 2.3. If $\mathcal{R}_{0}>1+k \Lambda / d$ and $0<r<1$ is such that $\frac{1}{\mathcal{R}_{0}}<\frac{r}{1+k \Lambda / d}<1$, then for any $\tau \in[0,1]$ there are no solutions $(S, I)$ of $(5)$ on $\partial U$.

Proof. First note that $\left(S_{0}, I_{0}\right)$ is the only solution of (5) on $\partial D$ for any $\tau \in[0,1]$. If $(S, I) \in \partial U$, then $(S, I) \notin \partial D$, so

$$
(S, I) \in D \text { and } S(t) \geq r(\Lambda / d), \forall t .
$$

The following inequality in $D$ is valid:

$$
(d+\epsilon+\mu+\gamma)(1+k \Lambda / d) \geq\left[(d+\epsilon+\mu)+\frac{\gamma}{1+\tau \alpha I^{n}}\right](1+\tau k I) .
$$

Multiplying by $(1+\tau k I) / I$ and integrating the second equation in (5) on the interval $[0, T]$, we have that

$$
\int_{0}^{T} \frac{I^{\prime}(1+\tau k I)}{I} d t+\int_{0}^{T}\left(\left[(d+\epsilon+\mu)+\frac{\gamma}{1+\tau \alpha I^{n}}\right](1+\tau k I)\right) d t=\int_{0}^{T} \beta S d t ;
$$

but $\int_{0}^{T} \frac{I^{\prime}(1+\tau k I)}{I} d t=0$ because $I$ is $T$-periodic; and using the inequalities (7) and (8) one gets

$$
(d+\epsilon+\mu+\gamma)(1+k \Lambda / d) \geq \frac{1}{T} \int_{0}^{T} \beta S d t \geq r(\Lambda / d) \bar{\beta} .
$$

Now, from our hypothesis

$$
d+\epsilon+\mu+\gamma \geq \bar{\beta} \frac{r(\Lambda / d)}{(1+k \Lambda / d)}>\bar{\beta}(\Lambda / d) \frac{1}{\mathcal{R}_{0}}=d+\epsilon+\mu+\gamma,
$$

which is a contradiction.

Proof of Theorem 2.1. First by Lemma 2.3 the homotopy (5) is admissible.

On the other hand, note that the initial system of our homotopy $G_{0}$ is the same as the ending system of the homotopy $F_{1}$ in (3). Thus, using the conclusions developed in [5] and our Proposition 2.2 we conclude that the degree of the system where $\tau=0$ in the fixed domain $U$ is nonzero, i.e., $\operatorname{deg}\left(G_{0}, U\right) \neq 0$.

Using the invariance of the Leray-Schauder degree under homotopy by Lemma 2.3, we obtain that $\operatorname{deg}\left(G_{1}, U\right) \neq 0$.

Therefore the system (2) admits a non-trivial periodic solution, which proves Theorem 2.1 . 
Example 2.4. In order to testify the validity of our results we consider an example whose form is as equation (1); to model the transmission rate of this infection the term $\beta(t)$ can be approximated by

$$
\beta(t)=b_{0}\left[1+b_{1} \cos (2 \pi(t+a))\right],
$$

where $b_{0}$ is the baseline transmission rate, $b_{1}$ is the relative seasonal forcing and a accounts for the time when the transmission rate is maximal [10]. This transmission rate assumes that the period of transmission is one year. We take $b_{0}=60, b_{1}=0.16$ and $a=0.15$. We also set $\Lambda=0.2, d=0.041, k=1, \mu=0.01, \epsilon=0.001, \gamma=10, n=10$ and $\alpha=2$ in system (1). We based some of these choices of parameters on the parameter ranges developed in [10], for the city of Gambia.

Numerical simulation are shown in the next figure which exhibits an oscillatory behavior for the susceptible and infected population according to the Theorem 2.1.

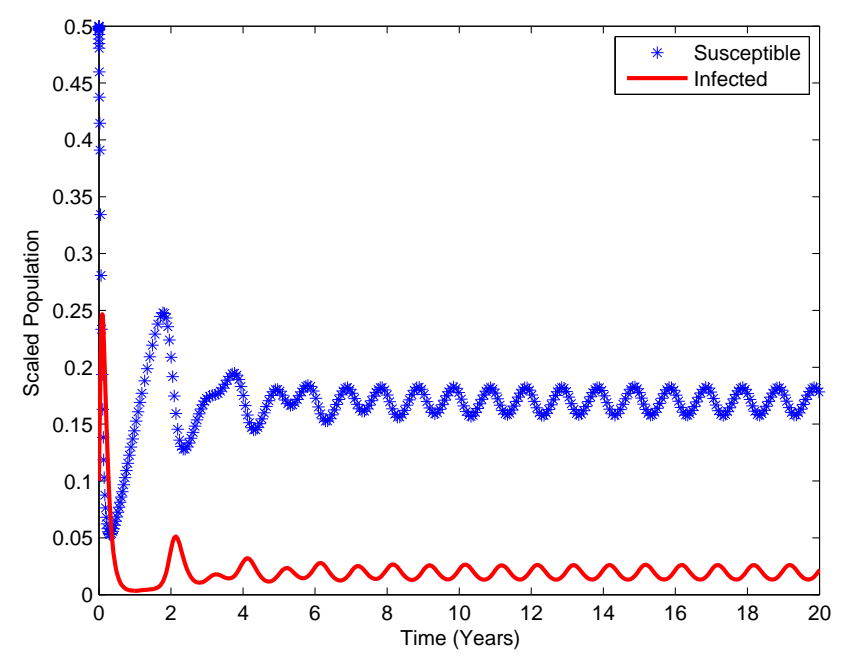

Figure 1. Existence of a periodic solution. The initial conditions are $S_{0}=0.5, I_{0}=0.1$ and $R_{0}=0.0$.

\section{Acknowledgements}

The authors would like to thank the anonymous referees for their helpful comments.

\section{References}

[1] Brown R.F., A topological introduction to nonlinear analysis, Second ed., Birkhäuser Boston, Inc., Boston, MA, 2004.

[2] Capasso V. and Serio G., "A generalization of the Kermack-McKendrick deterministic epidemic model", Math. Biosci. 42 (1978), No. 1-2, 43-61.

[3] Gaines R.E. and Mawhin J.L., Coincidence degree and nonlinear differential equations, Springer-Verlag, Berlin-New York, 1977.

[Revista Integración 
[4] González-Ramírez L.R., Osuna O. and Santaella-Forero R., "Periodic orbits for seasonal SIRS models with non-linear incidence rates", Electron. J. Differential Equations 2015 (2015), No. 300, 1-10.

[5] Katriel G., "Existence of periodic solutions for periodically forced SIR model", J. Math. Sci. (N.Y) 201 (2014), No. 3, 335-342.

[6] Li L., Bai Y. and Jin Z., "Periodic solutions of an epidemic model with saturated treatment", Nonlinear Dynam. 76 (2014), No. 2, 1099-1108.

[7] Liu W.M., Levin S.A. and Iwasa Y., "Influence of nonlinear incidence rates upon the behaviour of SIRS epidemiological models", J. Math. Biol. 23 (1986), No. 2, 187-204.

[8] Song B., Du W. and Lou J., "Different types of backward bifurcations due to densitydependent treatments", Math. Biosci. Eng. 10 (2013), No. 5-6, 1651-1668.

[9] van den Driessche P. and Watmough J., "Reproduction numbers and sub-threshold endemic equilibria for compartmental models of disease transmission", Math. Biosci. 180 (2002), 2948 .

[10] Weber A., Weber M. and Milligan P., "Modeling epidemics caused by respiratory synctial virus (RSV)", Math. Biosci. 172 (2001), No. 2, 95-113.

[11] Xiao D. and Ruan S., "Global analysis of an epidemic model with non monotone incidence rate", Math. Biosci. 208 (2007), No. 2, 419-429.

[12] Zhang X. and Liu X., "Backward bifurcation of an epidemic model with saturated treatment function", J. Math. Anal. Appl. 348 (2008), No. 1, 433-443.

Vol. 34, No. 2, 2016] 